\section{Sarcopenia definitions: where to draw the line? Response to Scarlata et al}

We thank Scarlata et $a l^{1}$ for their interest in our study ${ }^{2}$ in which we demonstrated a $15 \%$ prevalence in stable COPD by European Working Group on Sarcopenia in Older People (EWGSOP) criteria. $^{3}$ Professor Scarlata and colleagues acknowledge these are the most commonly adopted sarcopenia criteria. Consequently, prevalence estimates in other populations ${ }^{4}$ allow us to compare our findings beyond respiratory medicine. Towards the end of recruitment, The Foundation for the National Institutes of Health (FNIH) Sarcopenia Project published their findings. ${ }^{5}$ We acknowledge the strength of the
FNIH criteria, with cut points derived from a large pool of patient-level data. ${ }^{5}$ Nonetheless, the project team had to make difficult decisions around data harmonisation and handling, and by group's own admission the criteria are a 'work in progress' to be evolved and refined. ${ }^{6}$

Professor Scarlata and colleagues suggest the FNIH employ gait speed as an end point rather than a parameter of muscle function. However, low gait speed $(<0.8 \mathrm{~m} / \mathrm{s})$ was the principal criterion for mobility disability, from which grip strength and then appendicular lean mass cut points were derived. In this respect, we view gait speed as an intrinsic component of the FNIH operationalisation. ${ }^{5}$ With regards to lean mass assessment, the FNIH elected only to accept dual-energy X-ray absorptiometry (DXA) measurements and in doing so excluded over half of their data set (14 603/26 625 cases). While DXA offers a more accurate assessment of lean mass than bioelectrical impedance analysis, it may not be readily available in practice, particularly within settings where sarcopenia is relevant (nursing homes, critical care). In contrast, the EWGSOP criteria are more pragmatic and accept use of bioelectrical impedance analysis, a measure we routinely use in practice. ${ }^{7}$ Finally, when deciding between absolute and normalised cut points, FNIH data were inconsistent across genders; body mass index adjustment did not affect relationships between sarcopenia domains in men, but markedly improved the fit of models in women. ${ }^{5}$ Further, for all combinations of FNIH-defined low strength and mass, there were no significant associations with survival among women. ${ }^{6}$ This contrasts with recent studies in which EWGSOP-defined sarcopenia carried a twofold mortality risk. ${ }^{8}$

We are unable to offer a sarcopenia prevalence by FNIH criteria owing to lack of DXA measures in our cohort. $^{2}$ However, 2119 patients within the FNIH data set had a COPD diagnosis and disease-specific publications may emerge. ${ }^{5}$ We agree that multiple definitions may hinder efforts to treat sarcopenia. However, we are encouraged that a consistent phenotype is emerging in which a lean mass criterion is combined with one or more markers of physical function. This is important and exciting given the effectiveness of exercise, and nutritional and pharmacological interventions on functional end points. We observed a modest whole-group effect on all EWGSOP sarcopenia domains following pulmonary rehabilitation. ${ }^{2}$ To this end, patients falling close to a cut point before intervention had a reversal of their sarcopenia status. This phenomenon would have occurred irrespective of the cut points or definition used. We look forward to further interventional studies that capitalise on this shared feature of recent definitions, and reveal sarcopenia as a common but treatable problem.

Matthew Maddocks, ${ }_{1}^{1}$ Sarah E Jones, ${ }^{2}$
Samantha S C Kon, ${ }^{2}$ Jane L Canavan,
Claire M Nolan, ${ }^{2,3}$ Amy L Clark, Michael I Polkey, ${ }^{2}$ William D-C Man ${ }^{2,3}$

${ }^{1}$ King's College London, Cicely Saunders Institute, London, UK

${ }^{2}$ NIHR Respiratory Biomedical Research Unit, Royal Brompton \& Harefield NHS, Foundation Trust and Imperial College, London, UK

${ }^{3}$ Harefield Pulmonary Rehabilitation Unit, Royal Brompton and Harefield NHS Foundation Trust, London, UK

Correspondence to Dr Matthew Maddocks, King's College London, Cicely Saunders Institute, London SE5 9PJ, UK; matthew.maddocks@kcl.ac.uk

Competing interests None.

Provenance and peer review Not commissioned; internally peer reviewed.

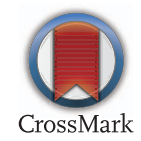

To cite Maddocks M, Jones SE, Kon S S C, et al. Thorax 2015;70:694.

Accepted 12 March 2015

Published Online First 8 April 2015

Thorax 2015;70:694.

doi:10.1136/thoraxjnl-2015-207041

\section{REFERENCES}

1 Scarlata S, Cesari M, Incalzi RA. Sarcopenia in COPD. Thorax 2015;70:693-4.

2 Jones SE, Maddocks M, Kon SS, et al. Sarcopenia in COPD: prevalence, clinical correlates and response to pulmonary rehabilitation. Thorax 2015;70:213-18.

3 Cruz-Jentoft AJ, Baeyens JP, Bauer JM, et al. Sarcopenia: European consensus on definition and diagnosis: Report of the European Working Group on Sarcopenia in Older People. Age Ageing 2010;39:412-23.

4 Cruz-Jentoft AJ, Landi F, Schneider SM, et al. Prevalence of and interventions for sarcopenia in ageing adults: a systematic review. Report of the International Sarcopenia Initiative (EWGSOP and IWGS). Age Ageing 2014;43:748-59.

5 Studenski SA, Peters KW, Alley DE, et al. The FNIH sarcopenia project: rationale, study description, conference recommendations, and final estimates. J Gerontol A Biol Sci Med Sci 2014;69:547-58.

6 Dam TT, Peters KW, Fragala M, et al. An evidence-based comparison of operational criteria for the presence of sarcopenia. J Gerontol A Biol Sci Med Sci 2014;69:584-90.

7 Maddocks M, Kon SS, Jones SE, et al. Bioelectrical impedance phase angle relates to function, disease severity and prognosis in stable chronic obstructive pulmonary disease. Clin Nutr 2015. pii: S0261-5614 (15)00003-5.

8 Arango-Lopera VE, Arroyo P, Gutierrez-Robledo LM, et al. Mortality as an adverse outcome of sarcopenia. J Nutr Health Aging 2013;17:259-62. 